\title{
An Examination of Serum Acylcarnitine and Amino Acid Profiles at Different Time Point of Ketogenic Diet Therapy and Their Association of Ketogenic Diet Effectiveness
}

\author{
Pi-Lien Hung ${ }^{1}$, Ju-Li Lin ${ }^{2}$, Chien Chen ${ }^{3}$, Kai-Yin Hung ${ }^{4}$, Tzu-Yun Hsieh ${ }^{5}$, Mei-Hsin Hsu ${ }^{1,5}$, Hsuan-Chang Kuo ${ }^{5}$ \\ and Ying-Jui Lin ${ }^{5, *}$ \\ 1 Department of Pediatrics, Division of Pediatric Neurology, Kaohsiung Chang Gung Memorial Hospital, \\ Chang Gung University College of Medicine, Kaohsiung 833, Taiwan; flora1402@cgmh.org.tw (P.-L.H.); \\ a03peggy@cgmh.org.tw (M.-H.H.) \\ 2 Department of Pediatrics, Division of Genetics and Endocrinology, Linkou Chang Gung Memorial Hospital, \\ Chang Gung University College of Medicine, Taoyan 333, Taiwan; lin001@cgmh.org.tw \\ 3 Department of Neurology, Taipei Veterans General Hospital, National Yang-Ming University, Taipei 112, \\ Taiwan; cchien0604@gmail.com \\ 4 Division of Nutritional Therapy, Kaohsiung Chang Gung Memorial Hospital, Kaohsiung 833, Taiwan; \\ redrosahung@cgmh.org.tw \\ 5 Department of Pediatrics, Division of Pediatric Critical Care, Kaohsiung Chang Gung Memorial Hospital, \\ Chang Gung University College of Medicine, Kaohsiung 833, Taiwan; role0302@cgmh.org.tw (T.-Y.H.); \\ kuohc117@cgmh.org.tw (H.-C.K.) \\ * Correspondence: rayray@cgmh.org.tw; Tel.: +886-7-731-7123 (ext. 8795); Fax: +886-7-733-8009
}

check for

updates

Citation: Hung, P.-L.; Lin, J.-L.; Chen, C.; Hung, K.-Y.; Hsieh, T.-Y.; Hsu, M.H.; Kuo, H.-C.; Lin, Y.-J. An Examination of Serum Acylcarnitine and Amino Acid Profiles at Different Time Point of Ketogenic Diet Therapy and Their Association of Ketogenic Diet Effectiveness. Nutrients 2021, 13, 21. https://dx.doi.org/10.3390/ nu13010021

Received: 22 November 2020 Accepted: 18 December 2020 Published: 23 December 2020

Publisher's Note: MDPI stays neutral with regard to jurisdictional claims in published maps and institutional affiliations.

Copyright: () 2020 by the authors. Licensee MDPI, Basel, Switzerland. This article is an open access article distributed under the terms and conditions of the Creative Commons Attribution (CC BY) license (https: / / creativecommons.org/ licenses/by/4.0/).

\begin{abstract}
Background: This study aimed to identify metabolic parameters at different time points of ketogenic diet therapy (KDT) and investigate their association with response to KDT in pediatric drug-resistant epilepsy (DRE). Methods: Prospectively, twenty-nine patients (0.67 20 years old) with DRE received classic ketogenic diet with non-fasting, gradual KD initiation protocol (GRAD-KD) for 1 year were enrolled. A total of 22 patients remaining in study received blood examinations at baseline, 3rd, 6 th, 9th, and 12th months of KDT. $\beta$-hydroxybutyrate, free carnitine, acylcarnitines, and amino acids were compared between responders (seizure reduction rate $\geq 50 \%$ ) and nonresponders (seizure reduction rate $<50 \%$ ) to identify the effectiveness of KDT. Results: The 12-month retention rate was $76 \%$. The responders after 12 months of KDT were $59 \%(13 / 22)$. The free carnitine level decreased significantly at 9 th months $(p<0.001)$ but increased toward baseline without symptoms. Propionyl carnitine (C3), Isovaleryl carnitine (C5), 3-Hydroxyisovalerylcarnitine (C5:OH) and methylmalonyl carnitine (C4-DC) decreased but 3-hydroxybutyrylcarnitine $(\mathrm{C} 4: \mathrm{OH})$ increased significantly at 12th months of KDT. The glycine level was persistently higher than baseline after KDT. KDT responders had lower baseline C3 and long-chain acylcarnitines, C14 and C18, as well as lower C5, C18, and leucine/isoleucine. Conclusions: KDT should be avoided in patients with non-ketotic hyperglycemia. Routine carnitine supplementation is not recommended because hypocarnitinemia was transient and asymptomatic during KDT. Better mitochondrial ßoxidation function associates with greater KDT response.
\end{abstract}

Keywords: ketogenic diet; free carnitine; acylcarnitines; amino acids; mitochondrial ßoxidation function

\section{Introduction}

Drug-resistant epilepsy (DRE) refers to epilepsy that does not respond to two or more antiepileptic drugs and accounts for $20-30 \%$ of childhood epilepsy. Thirty-three percent of patients with DRE who receive classic ketogenic diet therapy (KDT) have a more than $50 \%$ reduction in seizures and $15-20 \%$ become seizure-free [1-5]. KD is also thought to be effective in two metabolic disorders, i.e., glucose transporter type 1 deficiency syndrome, 
pyruvate dehydrogenase deficiency and in certain mitochondrial disorders [6]. However, with exception of the above metabolic and brain disorders, the response to KDT cannot be predicted.

Specific electroencephalography parameters had been reported to predict a favorable response to KDT in patients with DRE [7]. Biochemical parameters, including glucose and plasma $\beta$-hydroxybutyrate $(\beta \mathrm{HB})$ levels, the ratio of blood glucose to ketones, and the glucose ketone index had been reported to be associated with the efficacy of KDT in brain cancer in vivo [8]. Schoeler et al. reported a positive association between a higher baseline acetyl-carnitine level and greater efficacy of KDT [9]. However, there is limited evidence for an association between biochemical parameters and the effectiveness of KDT. Prolong KDT produces ketosis with increasing formation of acylcarnitines from free carnitine, leading to a drop in plasma free carnitine [10]. However, there were also scanty evidences regarding association between free carnitine and KD response.

There are several possible biochemical explanations for how KDT could control seizures. It had been reported that the antiepileptic effect of KDT results from a shift in the biogenesis of the mitochondria to a less hyperexcitable state. KDT restricts sugar and produces ketones that interact with receptors, channels, and enzymes [11-13]. Changes in neurotransmitter and amino acid levels in cerebrospinal fluid (CSF) in response to KDT may have anticonvulsant effects [14]. One report suggested that changes in the brain glutamate level brought about by KDT help to diminish seizures $[15,16]$. Furthermore, differences in CSF lysine and arginine levels had been found between responders and non-responders to KDT for epilepsy. Amino acids seem to play an important role in the antiepileptic effect of KDT; however, unlike for CSF, there are few reports on the changes in blood amino acid levels, in response to KDT [14]. Jirapinyo et al. found a high serum branched-chain amino acid/aromatic amino acid ratio after 10 days of KDT in a pediatric population [17]; however, the association between serum amino acids and the effectiveness of KDT is still under investigation. We hypothesized that some metabolic parameters at baseline or during KDT can predict or be associated with response to KDT. The aim of this study was to identify predictors and factors associated with the effectiveness of KDT in DRE.

\section{Materials and Methods}

\subsection{Participants}

Children with DRE were enrolled in this single-center, prospective, cohort study to receive a ketogenic diet from January 2018 to January 2019. The study was supervised by the Committee of Institutional Review Board at Chang Gung Memorial Hospital (IRB no.201700968A3). Informed consent was obtained from all guardians on enrollment.

DRE was defined as failure of adequate trials of two tolerated and appropriately used antiepileptic drug (AED) schedules (either as monotherapy or in combination) to achieve sustained seizure freedom [18]. Children with a confirmed diagnosis of inborn error of metabolism (pyruvate carboxylase deficiency, primary carnitine deficiency, fatty acid oxidation deficiency, ketolysis deficiency, familial hypercholesterolemia) and those who were surgical candidates were excluded.

\subsection{Study Protocol}

All enrolled patients were admitted to a pediatric general ward at Kaohsiung Chang Gung Memorial Hospital to receive a 5-day diet program. All study subjects underwent metabolic screening, including blood ammonia and lactate levels, serum cholesterol and triglyceride levels, urinary organic acids, and blood spot tests for amino acid profiles to exclude medical conditions that were inappropriate for KDT. All subjects were screened for a family history of porphyria during the initial visit. Classic ketogenic diet (cKD) was initiated in each patient with GRAD-KD protocol during the 5-day hospitalization. A registered dietitian calculated the energy requirement based on daily activity, dietary history, age, body weight and height. During the 5-day admission, patients received 
one-ninth of Recommended Dietary Allowance (RDA) calories as KD prescription on day 1 , one-sixth of RDA calories on day 2, one-third of RDA calories on day 3, two-thirds of RDA calories on day 4 , and the full RDA calories on day 5 . The keto ratio started at 2:1 on day 1 and gradually increased to $3 \sim 4: 1$ on day 5 , which approached $85 \%$ fat, $12 \%$ protein and $3 \%$ carbohydrate. The daily protein intake, predominantly animal protein, was in the range of $1.5-2.5 \mathrm{~g} / \mathrm{kg} /$ day. Medium chain triglyceride powder was prescribed gradually at a minimum dosage of $40 \mathrm{~g} /$ day if patients can tolerate it. Blood sugar and $\beta \mathrm{HB}$ levels were monitored every $2-4 \mathrm{~h}$ during the hospitalization period to avoid hypoglycemia and hyperketosis. The blood $\beta$ HB level was obtained from a fingertip blood sample (Free Style Optium Neo Blood Glucose and Ketone Monitoring System; Abbott Diabetes Care Inc., Whitney, UK) taken on the 2nd day of the 5-day diet program. On the day of discharge, the KDT nursery team educated the families on how to measure the urinary ketone level using a dipstick (Ketostix ${ }^{\circledR}$, Bayer Diabetes, Berkshire, UK). Seizure frequency and $\beta \mathrm{HB}$ level were recorded by seizure diaries at monthly follow-up visits for 12 months.

\subsection{Determination of Response to KDT}

The response to KDT was estimated in 28-day epochs for 12 months. Monthly seizure frequencies were calculated from seizure diaries. The formula used to calculate seizure reduction rate was $[(a-b) / a] \times 100$ where " $a$ " is the number of seizures in the 28-day baseline period and " $\mathrm{b}$ " is the number of seizures in the 28 days before the 12 -month follow-up visit [9]. Patients with a reduction in seizure frequency of $\geq 50 \%$ were defined as responders, and those with a reduction of $<50 \%$ were defined as non-responders.

\subsection{Tandem Mass Spectrometry}

Blood samples were taken after $10 \mathrm{~h}$ of fasting at baseline and at 3, 6, 9, and 12 months for measurement of fatty acids and amino acids by tandem mass spectrometry. The samples were prepared by removing the entire dried blood sample using a standard 0.3- $\mathrm{cm}$ singlehole punch into a 1-mL 96-well plate (Waters Corp., Milford, MA, USA; catalog number WAT058957). A 200- $\mu \mathrm{L}$ working standard (1:490 diluted in methanol; Cambridge Isotope Laboratories, Tewksbury, MA, USA; catalog numbers NSK-A and NSK-B) was placed in the 96-well plate. The plate incubated on a microplate shaker (Taitec, Tokyo, Japan) at a speed of 400-450 rpm. The supernatant was transferred to a Nunc plate (catalog number 249,944; Nunc, Roskilde, Denmark) and dried using a TurboVap (Caliper life Sciences, Hopkinton, MA, USA) at $70{ }^{\circ} \mathrm{C}$. Samples were reconstituted using $60 \mu \mathrm{L}$ of $80 \%$ deionized water in acetonitrile, mixed using the Wellmix on high speed for $1 \mathrm{~min}$. The mass analysis was performed using a Micromass Quattro Ultima Pt mass spectrometer (Waters 2795). Analyte-specific mass spectrometry parameters were optimized for the highest signal intensity. The data were analyzed using Masslynx 4.0 version software (Waters).

\subsection{Statistical Analysis}

The data are presented as the mean \pm standard error of the mean. Demographic parameters were examined by Fisher's exact test. Biochemical parameters, including $\beta \mathrm{HB}$ and plasma acylcarnitines [19] and amino acid levels were compared in and between groups at the various time points by conducting generalized estimating equation (GEE) after adjusting confounding effect of anticonvulsants. Associations between $\beta \mathrm{HB}$ levels, free carnitine levels, and seizure outcomes were analyzed by the Spearman zero order correlation coefficient. Receiver-operating characteristic (ROC) curve analysis was used to identify metabolites that are associated with the effectiveness of KDT. The area under the curve (AUC) values were determined using the receiver-operating characteristic ROC curve. The $95 \%$ confidence interval of area under curve (AUC) was produced by using the pROC [19] package in R (version 3.5.2; R Project for Statistical Computing, https: / / www.r-project.org). All statistical analysis was performed using SPSS for Windows version 19.0 software (IBM Corp.). $p<0.05$ was considered statistically significant. 


\section{Results}

\subsection{Clinical Characteristics}

Seven of the 29 patients who met the diagnostic criteria for DRE did not complete the 12-month study. These seven patients withdrew before completion of 9 months either due to diet intolerance or parent-related or child-related reasons. Twenty-two patients completed 12 months of KDT. The diet retention rate at 3, 6, 9, and 12 months were $89.6 \%, 83 \%, 76 \%$, and $76 \%$, respectively (Figure 1). The clinical characteristics of the 22 patients were shown in Table S1. The mean patient age was $9.3 \pm 1.98$ years (range, 8 months to 19 years), and the male to female ratio was 1.8:1. Generalized seizures occurred in $36.3 \%(8 / 22)$ of patients, focal seizures in $41 \%(9 / 22)$, and infantile spasm in $23 \%(5 / 22)$. The leading etiology was genetic $(31.8 \%, 7 / 22)$ followed by hypoxic-ischemic encephalopathy $(22.7 \%$, $5 / 22)$. Five $(22.7 \%, 5 / 22)$ of patients had a seizure reduction rate of $\leq 25 \%$, four $(22.7 \%$, $4 / 22)$ had a reduction of $25-50 \%$, nine had a reduction of $50 \%-90 \%$, and four $(18.2 \%, 4 / 22)$ had a reduction of $\geq 90 \%$. The response rate (seizure reduction rate $\geq 50 \%$ ) to 12 months of KDT was $59 \%(13 / 22)$, with three patients $(13.6 \%, 3 / 22)$ becoming seizure-free.

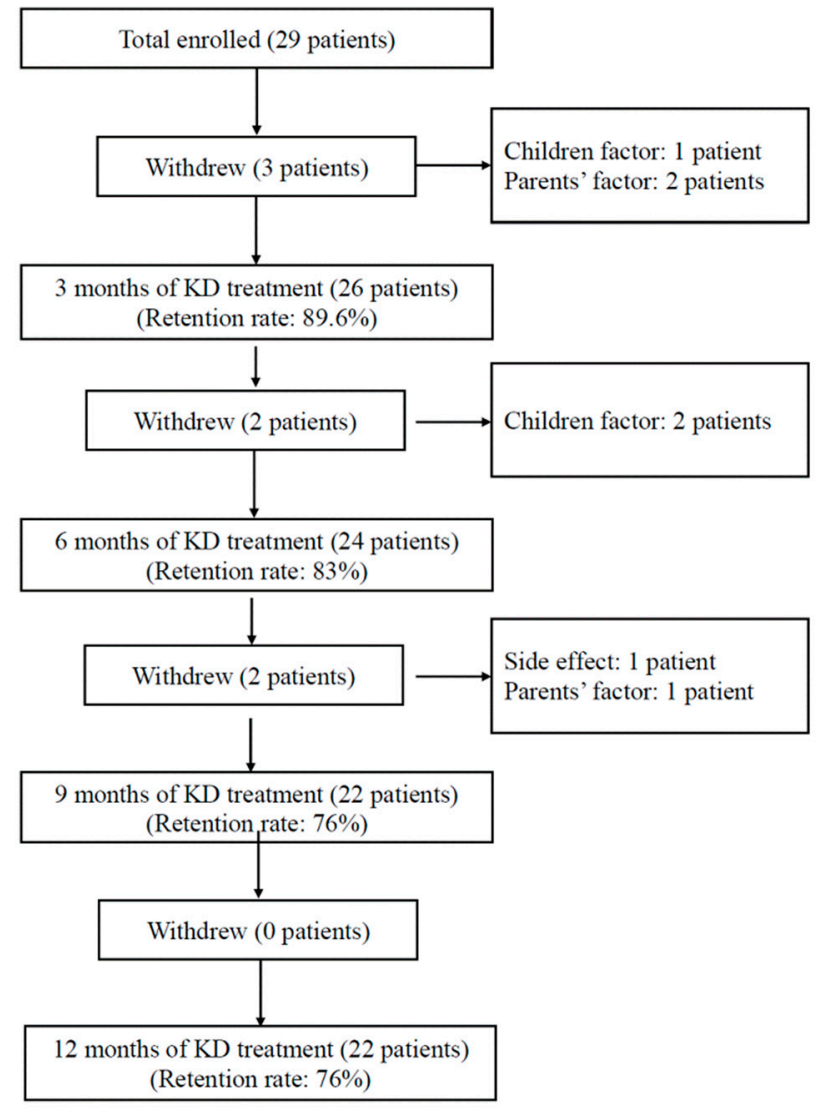

Figure 1. Flow chart showing the study protocol.

\subsection{Metabolic Changes after KDT}

All biochemical parameters were adjusted by GEE to exclude confounding effect of anticonvulsants (AEDs). Plasma acylcarnitines, and amino acids level at different time point of KDT were compared with those at baseline (Table 1). 



and 12 month of ketogenic diet therapy. All statistically significant values are bolded.

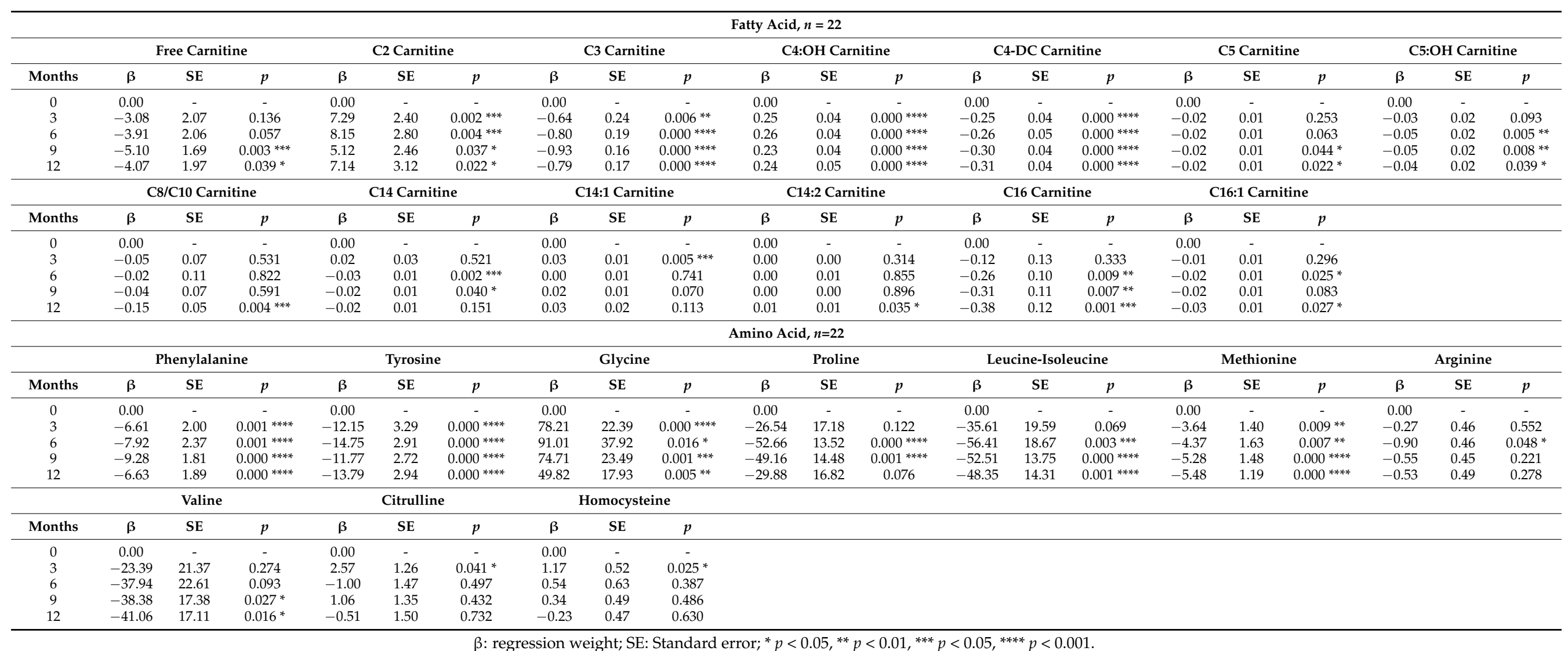




\subsection{Short-Chain Acylcarnitines after KDT}

Acetylcarnitine (C2), an important substrate for mitochondrial energy metabolism, was significantly higher than baseline level from 3 months to 12 months $(C 2, \beta=7.14$, $p<0.05)$. Propionate acyl-carnitine (C3) and methylmalonyl carnitine (C4-DC) levels were significantly lower than baseline value from 3 months to 12 months $(C 3, \beta=-0.79, p<0.001$; C4-DC, $\beta=-0.31, p<0.001)$. 3-hydroxybutyrylcarnitine $(\mathrm{C} 4: \mathrm{OH})$, derives from CoA-ester of the ketone body, D-3-hydroxybutyrate [20], was significantly higher than baseline value from 3 months to 12 months $(\mathrm{C} 4: \mathrm{OH}, \beta=0.24, p<0.001)$. Isovaleryl carnitine (C5) was significantly lower than baseline value from 9 months to 12 months (C5, $\beta=-0.02, p<0.05$ ). 3-Hydroxyisovalerylcarnitine $(\mathrm{C5}: \mathrm{OH})$ was significantly lower than baseline value from 6 months to 12 months (C5:OH, $\beta=-0.04, p<0.05)$. C3, C5, and $\mathrm{C} 5: \mathrm{OH}$ are degradation products of ketogenic amino acids: lysine, tryptophan, leucine and isoleucine [21].C4-DC is derived from branch-chain amino acids metabolism: leucine, isoleucine and valine which are ketogenic or glucogenic amino acids, showed a positive correlation of basal glucose level [22]. The results herein disclosed that KDT successfully led to ketogenesis by increasing CoA-ester formation from fatty acid $\beta$ oxidation instead of degrading ketogenic amino acids. On the other hand, increasing acetylcarnitine production in our results can explain how KDT could alter mitochondrial energy metabolism.

\subsection{Medium-Chain Acylcarnitines after KDT}

Octanoyl-(C8)/decanoyl-(C10) acyl-carnitine levels were significantly lower than baseline values at 12 months $(\mathrm{C} 8 / \mathrm{C} 10, \beta=-0.15, p<0.005)$. We supposed that the $\mathrm{C} 8 / \mathrm{C} 10$ ratio became lower after KDT may be due to highest net ketogenic effect of $\mathrm{C} 8$ over another medium-chain triglyceride [23] so that C8 was rapidly turn over into ketone body.

\subsection{Long-Chain Acylcarnitnes after KDT}

C14 carnitine was significantly lower than baseline values at 6 months and at 9 months; however, it became not significantly different from baseline value at 12 months (C14, $\beta=-0.03, p<0.005$ at 6 mons; $\beta=-0.02, p<0.05$ at 9 months). C14:1 was transiently higher than baseline values at 3 months (C14:1, $\beta=0.03, p<0.01)$. C14:2 carnitine was significantly higher than baseline values at 12 months (C14:2, $\beta=0.01, p<0.05)$. C16 carnitine was significantly lower than baseline values from 6 months to 12 months (C16, $\beta=-0.26, p<0.005$ at 6 months; $\beta=-0.31, p<0.01$ at 9 months; $\beta=-0.38, p<0.01$ at 12 months). C16:1 carnitine was significantly lower than baseline values at 6 months and 12 months (C16:1, $\beta=-0.02, p<0.05$ at 6 months; $\beta=-0.03, p<0.05$ at 12 months). C18 carnitine showed no significant changes after KDT. Our data demonstrated that C14:1 and C14:2 carnitine levels trended upwards during the study period.

\subsection{Amino Acids after KDT}

After 12 months of KDT, there was a significant decrease in levels of ketogenic amino acids, including phenylalanine, tyrosine, and leucine/isoleucine (Phenylalanine, $\beta=-6.63$, $p<0.001$; tyrosine, $\beta=-13.79, p<0.001$; leucine/isoleucine, $\beta=-48.35, p<0.001$ ). Methionine, a substrate for biogenesis of carnitine, was significantly lower than baseline value from 3 months to 12 months of KDT (Methionine, $\beta=-5.48, p<0.001$ ). It shared the similar tendency of free carnitine in our study. Glycine was the unique amino acid which increased its level significantly with compared to baseline levels from starting KD to 12 months of KDT (Glycine, $\beta=78.21, p<0.001$ at baseline; $\beta=91.01, p<0.05$ at 3 months; $\beta=74.71, p<0.005$ at 6 months; $\beta=49.82, p<0.01$ at 12 months). The glucogenic amino acid, valine, showed significantly lower than baseline values at 9 months and 12 months of KDT (valine, $\beta=-38.38, p<0.05$ at 9 months; $\beta=-41.06, p<0.05$ at 12 months). In addition, proline was transiently lower than baseline at 6 months and 9 months (proline, $\beta=-52.66, p<0.001$ at 6 months; $\beta=-49.16, p<0.005$ at 12 months), but it increased at 12 months and showed no significant differences compared to baseline values. Arginine was transiently lower at 6 months, whereas citrulline and homocysteine were transiently 
higher at 3 months (arginine, $\beta=-0.90, p<0.05$ at 3 months; citrulline, $\beta=2.57, p<0.05$ at 3 months; homocysteine, $\beta=1.17, p<0.05$ at 3 months). However, the serum level of these three amino acids showed no significant changes compared to baseline values at 12 months of KDT. Our results demonstrated that the concentrations of ketogenic amino acids and some glucogenic amino acids became significantly lower after 12 months of KDT. In addition, glycine was found persistently higher than baseline throughout the KDT period.

\subsection{Free Carnitine after KDT}

There was a significant decrease in the free carnitine level after 9 months of KDT, and never returned to baseline level at the end of study (free carnitine, $\beta=-5.10, p<0.01$ at 9 months; $\beta=-4.07, p<0.05$ at 12 months). However, none of our study subjects had symptoms of hypocarnitinemia, such as muscle weakness, fatigue, or anorexia. None of our study subjects received carnitine supplement during the KDT.

\subsection{Differences in Metabolic Parameters between Responders and Non-Responders}

Demographic variables and clinical characteristics were compared between responders and non-responders at the different time points in Supplementary Tables S1 and S2. There was no significant between-group difference in type of seizure, etiology of epilepsy, body weight, and numbers of AEDs administered. Non-responders received more valproic acid (VPA) as monotherapy or adjuvant therapy than responders did (6 vs. $2, p<0.05$ ).

We offered a comparison of acylcarnitines and amino acids level between responders and non-responders as shown in Table 2. All the comparisons were examined by conducting GEE after adjusting the confounding effect of anticonvulsants. The results were demonstrated in Table 2 and were summarized as following paragraphs.

Table 2. Comparison of fatty acid acylcarnitines between responders and non-responders. Acylcarnitines profile of responders $(n=12)$ and non-responders $(n=10)$. Group differences were analyzed by conducting generalized estimating equation (GEE) after adjusting confounding effect of anticonvulsants. $R$ : responders; NR: non-responders.

\begin{tabular}{|c|c|c|c|c|c|c|c|c|c|}
\hline & $\begin{array}{c}\text { Time } \\
\text { (Month) }\end{array}$ & $\beta$ & SE & $p$ & & $\begin{array}{c}\text { Time } \\
\text { (Month) }\end{array}$ & $\beta$ & SE & $p$ \\
\hline \multirow[t]{5}{*}{ BHB } & 0 & 0.37 & 0.87 & 0.673 & \multirow{5}{*}{$\begin{array}{l}\text { C6-DC } \\
\text { carnitine }\end{array}$} & 0 & 0.01 & 0.01 & 0.102 \\
\hline & 3 & 0.31 & 0.57 & 0.588 & & 3 & 0.00 & 0.00 & 0.324 \\
\hline & 6 & 1.43 & 0.64 & $0.025 *$ & & 6 & -0.01 & 0.01 & $0.031 *$ \\
\hline & 9 & -0.65 & 0.74 & 0.384 & & 9 & -0.01 & 0.00 & 0.275 \\
\hline & 12 & -0.06 & 0.77 & 0.934 & & 12 & 0.00 & 0.00 & 0.465 \\
\hline \multirow[t]{5}{*}{$\begin{array}{c}\text { Free } \\
\text { carnitine }\end{array}$} & 0 & -7.85 & 3.73 & $0.035 *$ & \multirow[t]{5}{*}{$\begin{array}{c}\text { C8 } \\
\text { carnitine }\end{array}$} & 0 & -0.01 & 0.03 & 0.657 \\
\hline & 3 & -11.84 & 4.05 & $0.003^{* * *}$ & & 3 & -0.07 & 0.07 & 0.333 \\
\hline & 6 & -15.94 & 4.18 & $0.000^{* * * *}$ & & 6 & 0.01 & 0.01 & 0.405 \\
\hline & 9 & -6.77 & 3.58 & 0.059 & & 9 & -0.06 & 0.01 & $0.000^{* * * *}$ \\
\hline & 12 & -8.59 & 3.11 & $0.006^{* *}$ & & 12 & -0.01 & 0.01 & 0.230 \\
\hline \multirow[t]{5}{*}{$\begin{array}{c}\text { C2 } \\
\text { carnitine }\end{array}$} & 0 & -8.95 & 3.29 & $0.006^{* *}$ & \multirow[t]{5}{*}{$\begin{array}{c}\text { C10 } \\
\text { carnitine }\end{array}$} & 0 & -0.02 & 0.04 & 0.688 \\
\hline & 3 & -9.67 & 3.84 & $0.012 *$ & & 3 & -0.06 & 0.05 & 0.240 \\
\hline & 6 & -2.65 & 4.50 & 0.556 & & 6 & 0.02 & 0.02 & 0.333 \\
\hline & 9 & -1.60 & 4.96 & 0.747 & & 9 & -0.07 & 0.02 & $0.000^{* * * *}$ \\
\hline & 12 & 1.464 & 5.3079 & 0.783 & & 12 & -0.03 & 0.02 & 0.112 \\
\hline
\end{tabular}


Table 2. Cont

\begin{tabular}{|c|c|c|c|c|c|c|c|c|c|}
\hline & $\begin{array}{c}\text { Time } \\
\text { (Month) }\end{array}$ & $\beta$ & SE & $p$ & & $\begin{array}{c}\text { Time } \\
\text { (Month) }\end{array}$ & $\beta$ & $\mathrm{SE}$ & $p$ \\
\hline \multirow[t]{5}{*}{$\begin{array}{c}\text { C3 } \\
\text { carnitine }\end{array}$} & 0 & -1.28 & 0.60 & $0.032 *$ & \multirow[t]{5}{*}{$\begin{array}{c}\text { C10:1 } \\
\text { carnitine }\end{array}$} & 0 & 0.01 & 0.03 & 0.664 \\
\hline & 3 & -1.68 & 0.34 & $0.000^{* * * *}$ & & 3 & 0.02 & 0.02 & 0.355 \\
\hline & 6 & -1.80 & 0.30 & $0.000^{* * * *}$ & & 6 & 0.02 & 0.02 & 0.144 \\
\hline & 9 & -1.12 & 0.51 & $0.027 *$ & & 9 & -0.08 & 0.01 & $0.000^{* * * *}$ \\
\hline & 12 & -0.98 & 0.43 & $0.022 *$ & & 12 & -0.02 & 0.02 & 0.253 \\
\hline \multirow[t]{5}{*}{$\begin{array}{c}\text { C4 } \\
\text { carnitine }\end{array}$} & 0 & -0.05 & 0.06 & 0.456 & \multirow[t]{5}{*}{$\begin{array}{l}\mathrm{C} 8 / \mathrm{C} 10 \\
\text { carnitine }\end{array}$} & 0 & -0.04 & 0.17 & 0.817 \\
\hline & 3 & -0.21 & 0.06 & $0.000^{* * * *}$ & & 3 & 0.07 & 0.15 & 0.628 \\
\hline & 6 & -0.14 & 0.06 & 0.028 * & & 6 & -0.02 & 0.11 & 0.846 \\
\hline & 9 & -0.10 & 0.06 & 0.084 & & 9 & 0.07 & 0.13 & 0.575 \\
\hline & 12 & -0.03 & 0.05 & 0.547 & & 12 & -0.01 & 0.12 & 0.949 \\
\hline \multirow{5}{*}{$\begin{array}{c}\mathrm{C} 4: \mathrm{OH} \\
\text { carnitine }\end{array}$} & 0 & -0.07 & 0.03 & 0.033 * & \multirow[t]{5}{*}{$\begin{array}{c}\mathrm{C} 12 \\
\text { carnitine }\end{array}$} & 0 & -0.02 & 0.02 & 0.337 \\
\hline & 3 & -0.04 & 0.08 & 0.617 & & 3 & -0.07 & 0.03 & $0.027^{*}$ \\
\hline & 6 & 0.06 & 0.07 & 0.404 & & 6 & -0.02 & 0.01 & 0.151 \\
\hline & 9 & -0.07 & 0.08 & 0.379 & & 9 & -0.03 & 0.02 & 0.055 \\
\hline & 12 & 0.09 & 0.11 & 0.409 & & 12 & -0.01 & 0.02 & 0.560 \\
\hline \multirow[t]{5}{*}{$\begin{array}{c}\text { C4-DC } \\
\text { carnitine }\end{array}$} & 0 & -0.15 & 0.09 & 0.108 & \multirow[t]{5}{*}{$\begin{array}{c}\text { C14 } \\
\text { carnitine }\end{array}$} & 0 & -0.04 & 0.02 & $0.022 *$ \\
\hline & 3 & -0.16 & 0.08 & $0.043 *$ & & 3 & -0.11 & 0.08 & 0.146 \\
\hline & 6 & -0.06 & 0.10 & 0.500 & & 6 & -0.04 & 0.01 & $0.007 * *$ \\
\hline & 9 & 0.05 & 0.08 & 0.550 & & 9 & -0.04 & 0.02 & 0.016 * \\
\hline & 12 & 0.04 & 0.11 & 0.712 & & 12 & 0.00 & 0.01 & 0.979 \\
\hline \multirow[t]{5}{*}{$\begin{array}{c}\text { C5 } \\
\text { carnitine }\end{array}$} & 0 & -0.02 & 0.04 & 0.592 & \multirow[t]{5}{*}{$\begin{array}{c}\text { C14:1 } \\
\text { carnitine }\end{array}$} & 0 & -0.04 & 0.01 & $0.000^{* * * *}$ \\
\hline & 3 & -0.09 & 0.02 & $0.000^{* * * *}$ & & 3 & -0.05 & 0.02 & $0.022 *$ \\
\hline & 6 & -0.10 & 0.01 & $0.000^{* * * *}$ & & 6 & 0.00 & 0.02 & 0.911 \\
\hline & 9 & -0.07 & 0.02 & $0.002^{* * *}$ & & 9 & -0.05 & 0.02 & $0.009^{* *}$ \\
\hline & 12 & -0.04 & 0.01 & $0.002^{* * *}$ & & 12 & -0.01 & 0.02 & 0.564 \\
\hline \multirow[t]{5}{*}{$\begin{array}{c}\text { C5:1 } \\
\text { carnitine }\end{array}$} & 0 & 0.00 & 0.00 & 0.275 & \multirow[t]{5}{*}{$\begin{array}{c}\text { C14:2 } \\
\text { carnitine }\end{array}$} & 0 & 0.00 & 0.01 & 0.896 \\
\hline & 3 & -0.01 & 0.00 & 0.013 * & & 3 & -0.01 & 0.01 & 0.153 \\
\hline & 6 & 0.00 & 0.00 & 0.401 & & 6 & 0.00 & 0.01 & 0.831 \\
\hline & 9 & -0.01 & 0.00 & $0.001^{* * *}$ & & 9 & -0.03 & 0.01 & $0.003^{* * *}$ \\
\hline & 12 & 0.00 & 0.00 & 0.504 & & 12 & 0.00 & 0.01 & 0.761 \\
\hline \multirow[t]{5}{*}{$\begin{array}{c}\mathrm{C} 5: \mathrm{OH} \\
\text { carnitine }\end{array}$} & 0 & -0.08 & 0.09 & 0.371 & \multirow[t]{5}{*}{$\begin{array}{c}\text { C16 } \\
\text { carnitine }\end{array}$} & 0 & -0.70 & 0.25 & $0.005^{* *}$ \\
\hline & 3 & -0.12 & 0.04 & $0.006^{* *}$ & & 3 & -1.08 & 0.35 & $0.002^{* * *}$ \\
\hline & 6 & -0.10 & 0.05 & 0.062 & & 6 & -0.83 & 0.23 & $0.000^{* * * *}$ \\
\hline & 9 & -0.11 & 0.04 & 0.012 * & & 9 & -0.62 & 0.21 & $0.003^{* * *}$ \\
\hline & 12 & -0.11 & 0.09 & 0.224 & & 12 & -0.39 & 0.16 & $0.013^{*}$ \\
\hline
\end{tabular}


Table 2. Cont.

\begin{tabular}{|c|c|c|c|c|c|c|c|c|c|}
\hline & $\begin{array}{c}\text { Time } \\
\text { (Month) }\end{array}$ & $\beta$ & $\mathrm{SE}$ & $p$ & & $\begin{array}{c}\text { Time } \\
\text { (Month) }\end{array}$ & $\beta$ & SE & $p$ \\
\hline \multirow{5}{*}{$\begin{array}{l}\text { C5-DC } \\
\text { carnitine }\end{array}$} & 0 & 0.01 & 0.01 & 0.055 & \multirow{5}{*}{$\begin{array}{c}\text { C16:1 } \\
\text { carnitine }\end{array}$} & 0 & -0.07 & 0.03 & 0.011 * \\
\hline & 3 & -0.01 & 0.00 & $0.003^{* * *}$ & & 3 & -0.07 & 0.01 & $0.000^{* * * *}$ \\
\hline & 6 & 0.00 & 0.00 & 0.232 & & 6 & -0.05 & 0.02 & 0.014 * \\
\hline & 9 & -0.02 & 0.01 & 0.011 * & & 9 & -0.05 & 0.03 & 0.059 \\
\hline & 12 & -0.01 & 0.00 & $0.005^{* * *}$ & & 12 & -0.01 & 0.01 & 0.235 \\
\hline \multirow{5}{*}{$\begin{array}{c}\text { C6 } \\
\text { carnitine }\end{array}$} & 0 & -0.01 & 0.02 & 0.620 & \multirow{5}{*}{$\begin{array}{c}\text { C18 } \\
\text { carnitine }\end{array}$} & 0 & -0.47 & 0.08 & $0.000^{* * * *}$ \\
\hline & 3 & -0.09 & 0.06 & 0.152 & & 3 & -0.50 & 0.30 & 0.093 \\
\hline & 6 & -0.02 & 0.00 & $0.000^{* * * *}$ & & 6 & -0.51 & 0.18 & $0.004^{* * *}$ \\
\hline & 9 & -0.02 & 0.01 & $0.031 *$ & & 9 & -0.29 & 0.11 & $0.006^{* *}$ \\
\hline & 12 & 0.00 & 0.01 & 0.836 & & 12 & -0.22 & 0.16 & 0.168 \\
\hline
\end{tabular}

$\beta$ : regression weight; SE: Standard error; ${ }^{*} p<0.05,{ }^{* *} p<0.01,{ }^{* * *} p<0.05,{ }^{* * * *} p<0.001$.

\subsection{Free Carnitine and $\beta H B$ Levels in Responders}

There was a significant increase in the $\beta \mathrm{HB}$ level in responders at 6 months $(\beta \mathrm{HB}$, $\beta=1.43, p<0.05$ at 6 months). Free carnitine level in responders was significantly lower than that in non-responders after initiating of KDT (Free carnitine, $\beta=-7.85, p<0.05$ at baseline; $\beta=-11.84, p<0.005$ at 3 months; $\beta=-15.94, p<0.001$ at 9 months; $\beta=-6.77$, $p<0.05$ at 9 months; $\beta=-8.59, p<0.01$ at 12 months). Free carnitine levels reached a nadir in responders at 6 months (Supplementary Table S3, responders vs. non-responders, $20.84 \pm 6.89$ vs. $29.03 \pm 8.86, p<0.05)$, approaching the diagnostic criteria of carnitine deficiency defined as carnitine level $\leq 20 \mu \mathrm{M}$ or a ratio of acylated-to-free carnitine of $\geq 0.4$, at an age over 1 week post term [24], and gradually increased its level thereafter. There was a negative correlation between $\beta \mathrm{HB}$ and free carnitine levels (Supplementary Figure S1A, $\rho=-0.686, p<0.05)$. Carnitine is a crucial factor in the transfer of fatty acids for mitochondrial $\beta$-oxidation, and $\beta \mathrm{HB}$ is the major product of ketosis, we examined the $\beta \mathrm{HB}$ and free carnitine levels at 6 months to assess the association with efficacy of diet ( $\geq 50 \%$ seizure reduction). However, neither free carnitine nor $\beta \mathrm{HB}$ at 6 months had clear association with the efficacy of KDT (Supplementary Figure S1B, correlation coefficient of free carnitine and seizure reduction rate, $\rho=-0.109, p=0.781$; correlation coefficient of $\beta \mathrm{HB}$ and seizure reduction rate, $\rho=-0.427, p=0.252$ ).

\subsection{Short-Chain Acylcarnitines in Responders}

There was a decrease in $\mathrm{C} 2$ in responders at baseline and at 3 months, however the $\mathrm{C} 2$ levels became comparable between groups after 3 months (C2, $\beta=-8.95, p<0.005$ at baseline; $\beta=-9.67, p<0.05)$. C3 levels in responders were significantly lower after initiating of KDT to the end of study ( $\mathrm{C} 3, \beta=-1.28, p<0.05$ at baseline; $\beta=-1.68$, $p<0.001$ at 3 months; $\beta=-1.8, p<0.001$ at 6 months; $\beta=-1.12, p<0.05$ at 9 months; $\beta=-0.98, p<0.05$ at 12 months). $\mathrm{C} 4: \mathrm{OH}$ levels were significantly lower in responders than in non-responders at baseline, but the level became comparable between groups after initiating of KDT (C4:OH, $\beta=-0.07, p<0.05)$. C4-DC levels in responders were transiently and significantly lower at 3 months, and the levels became comparable between groups from 3 months to 12 month (C4-DC, $\beta=-0.06, p<0.05$ at 3 months). C5 levels in responders were significantly lower than that in non-responders from 3 to 12 months ( $C 5$, $\beta=-0.09, p<0.001$ at 3 months; $\beta=-0.10, p<0.001$ at 6 months; $\beta=-0.07, p<0.005$ at 9 months; $\beta=-0.04, p<0.005$ at 12 months). C5:1 level was fluctuating in responders. C5:1 levels were significantly lower in responders at 3 months and 9 months, but they showed no significant differences between groups at baseline, 6 months, and 12 months (C5:1, $\beta=-0.01, p<0.05$ at 3 months; $\beta=-0.01, p<0.05$ at 9 months). C5-DC levels were 
significantly lower in responders at 3 months, 9 months, and 12 months (C5-DC, $\beta=-0.09$, $p<0.001$ at 3 months; $\beta=-0.02, p<0.05$ at 9 months; $\beta=-0.01, p<0.01$ at 12 months). Our data demonstrated that $C 3$ and $C 5$ carnitines had persistently lower levels in responders than in non-responders after initiating of KDT.

\subsection{Medium-Chain Acylcarnitines in Responders}

We found C6 levels were significantly lower in responders at 6 months and 9 months, but there were no between-group differences at other time point $(\mathrm{C} 6, \beta=-0.02, p<0.001$ at 6 months; $\beta=-0.02, p<0.05$ at 9 months). C6-DC levels were significantly lower in responders only at 6 months, but there were no significant between-group differences at other time point (C6-DC, $\beta=-0.01, p<0.05$ at 6 months). C8, C10, and C10:1 levels were significantly lower in responders at 9 months, but there were no significant between-group differences at other time point ( $\beta$ and $p$ value at 9 months were shown as follows: $C 8$, $\beta=-0.06, p<0.001 ; \mathrm{C} 10, \beta=-0.07, p<0.001 ; \mathrm{C} 10: 1, \beta=-0.08, p<0.001)$. C12 levels were significantly lower in responders only at 3 months, but not at other time points (C12, $\beta=-0.07, p<0.05)$. In brief summary, although medium-chain acylcarnitines showed significantly lower in responders at some time points of KDT; however, none of them showed significant differences between responders and non-responders at endpoint of the study.

\subsection{Long-Chain Acylcarnitines in Responders}

C14 levels in responders were also significantly lower than that in non-responders at baseline, 6 months and 9 months $(\mathrm{C} 14, \beta=-0.04, p<0.05$ at baseline; $\beta=-0.04$, $p<0.01$ at 6 months; $\beta=-0.04, p<0.05$ at 9 months). C14:1levels were significantly lower in responders at baseline, 3 months and 9 months (C14:1, $\beta=-0.04, p<0.001$ at baseline; $\beta=-0.05, p<0.05$ at 3 months; $\beta=-0.05, p<0.01$ at 9 months). C14:2 levels were significantly lower in responders at 9 months, but there were no between-group significant differences at other time points ( $\beta=-0.03, p<0.005$ at 9 months). However, both C14:1 and C14:2 levels showed no significant between-group differences at 12 months. C16 levels were significantly lower in responders from initiating of KDT to the end of study (C16, $\beta=-0.70, p<0.01$ at baseline; $\beta=-1.08, p<0.005$ at 3 months; $\beta=-0.83$, $p<0.001$ at 9 months; $\beta=-0.39, p<0.05$ at 12 months). C16:1 levels were significantly lower in responders at baseline, 3 months and 9 months (C16:1, $\beta=-0.07, p<0.05$ at baseline; $\beta=-0.07, p<0.001$ at 3 months; $\beta=-0.05, p<0.05$ at 6 months). $C 18$ levels were significantly lower in responders at baseline, 6 and 9 months $(\mathrm{C} 18, \beta=-0.47, p<0.001$ at baseline; $\beta=-0.51, p<0.005$ at 6 months; $\beta=-0.29, p<0.01$ at 9 months). However, C18 levels showed no significant between-group differences at the endpoint of study. Our data disclosed that although blood levels of some long-chain acylcarnitines were significantly lower in responders at some time points of KDT; however, just palmitoyl carnitine (C16) levels were found significantly lower in responders at the endpoint of study.

\subsection{Amino Acids in Responders}

The comparison of amino acids between responders and non-responders was demonstrated in Table 3. Phenylalanine decreased in responders, reaching nadir levels at 6 months, and was significantly lower than in non-responders at 6 months and 9 months, but it tended to increase thereafter, although not to the baseline values by the end of the study (Phenylalanine, $\beta=-11.97, p<0.005$ at 6 months; $\beta=-3.81, p<0.05$ at 9 months). Levels of glycine increased significantly in responders from 3 months to 9 months, at which time they were significantly higher than the levels in non-responders (Glycine, $\beta=92.61, p<0.001$ at 3 months; $\beta=154.21, p<0.01$ at 6 months; $\beta=77.63, p<0.05$ at 9 months). Leucine/isoleucine levels in responders were significantly lower than that in non-responders at 3,9 , and 12 months of KDT (Leucine/isoleucine, $\beta=-58.47, p<0.001$ at 3 months; $\beta=-37.16, p<0.001$ at 9 months; $\beta=-36.19, p<0.01$ at 12 months). Phenylalanine/tyrosine ratio in responders was significantly lower than that in non-responders at 6 months and higher than that in 
non-responders at 12 months (phenylalanine/tyrosine ratio, $\beta=-0.3, p<0.001$ at 6 months; $\beta=0.21, p<0.001$ at 12 months). Arginine levels were significantly higher in responders at 6 months but not other time points (Arginine, $\beta=0.83, p<0.05$ at 6 months). Valine levels were significantly lower in responders at 9 months, but not at other time points (valine, $\beta=-21.57, p<0.05$ at 9 months). Ornithine levels were significantly higher in responders at baseline (ornithine, $\beta=9.72, p<0.05$ at baseline), but it got paradoxically lower than that in non-responders at 3 months and 6 months $(\beta=-7.56, p<0.01$ at 3 months; $\beta=-5.79$, $p<0.05$ at 6 months). Amino acids, including tyrosine, proline, methionine, glutamic acid, citrulline, and homocysteine showed no significant between-group differences at different time points of KDT. Our results demonstrated that leucine/isoleucine levels, the ketogenic amino acids, in responders were significantly lower at the endpoint of study, which may indicate that degrading ketogenic amino acids into acetyl-CoA significantly decreased in responders at 12 months of KDT. Although phenylalanine/tyrosine ratio was significantly higher in responders at 12 months, the mean phenylalanine level in responders never reached $\geq 400 \mathrm{umol} / 1$ and the ratio never reached $\geq 1.5$ at 12 months (mean phenylalanine level: $39.33 \pm 6.85 \mathrm{umoL} / \mathrm{L}$; mean ratio $1.14 \pm 0.24$ ). The relative high phenylalanine/tyrosine ratio in responders was presumptive no clinical significance.

Table 3. Comparison of amino acids between responders and non-responders. Amino acids profile of responders $(n=12)$ and non-responders $(n=10)$. Group differences were analyzed by GEE after adjusting confounding effect of anticonvulsants. $R$ : responders; $N R$ : non-responders.

\begin{tabular}{cccccccccc}
\hline & $\begin{array}{c}\text { Time } \\
\text { (month) }\end{array}$ & $\boldsymbol{\beta}$ & SE & $\boldsymbol{p}$ & & $\begin{array}{c}\text { Time } \\
\text { (month) }\end{array}$ & $\boldsymbol{\beta}$ & SE & $p$ \\
\hline Phenylalanine & 0 & 0.18 & 3.99 & 0.964 & Arginine & 0 & 1.52 & 1.06 & 0.154 \\
& 3 & -3.35 & 1.78 & 0.060 & & 3 & 0.63 & 1.08 & 0.560 \\
& 6 & -11.94 & 3.74 & $0.001^{* * *}$ & & 6 & 0.83 & 0.32 & $0.010^{* *}$ \\
& 9 & -3.81 & 1.63 & $0.019^{*}$ & & 9 & 0.83 & 0.76 & 0.272 \\
& 12 & 0.21 & 3.00 & 0.943 & & 12 & 0.74 & 0.46 & 0.107 \\
\hline Tyrosine & 0 & 4.84 & 7.65 & 0.527 & Phenylalanine & 0 & -0.04 & 0.09 & 0.618 \\
& 3 & -4.09 & 5.68 & 0.472 & /Tyrosine & 3 & -0.06 & 0.18 & 0.719 \\
& 6 & -0.99 & 2.38 & 0.677 & & 6 & -0.30 & 0.06 & $0.000^{* * * *}$ \\
& 9 & 1.38 & 4.20 & 0.743 & & 9 & -0.12 & 0.08 & 0.144 \\
Glycine & 12 & -7.21 & 3.77 & 0.056 & & 12 & 0.21 & 0.06 & $0.000^{* * * *}$ \\
& 0 & 38.49 & 25.29 & 0.128 & Alanine & 0 & -27.17 & 44.74 & 0.544 \\
& 3 & 92.61 & 25.09 & $0.000^{* * * *}$ & & 3 & -13.96 & 38.93 & 0.720 \\
& 6 & 154.21 & 58.87 & $0.009 * *$ & & 6 & -13.97 & 32.42 & 0.666 \\
& 9 & 77.63 & 38.82 & $0.046^{*}$ & & 9 & 15.19 & 21.28 & 0.475 \\
Proline & 12 & 53.38 & 33.84 & 0.115 & & 12 & 28.74 & 25.08 & 0.252 \\
\hline & 0 & -1.84 & 33.52 & 0.956 & Valine & 0 & -8.54 & 29.85 & 0.775 \\
& 3 & -0.04 & 31.20 & 0.999 & & 3 & -23.79 & 20.00 & 0.234 \\
& 6 & -7.89 & 21.46 & 0.713 & & 6 & -12.92 & 15.43 & 0.402 \\
& 9 & -23.27 & 15.74 & 0.139 & & 9 & -21.57 & 10.83 & $0.046^{*}$ \\
Leucine & 12 & -37.73 & 30.29 & 0.213 & & 12 & -21.74 & 18.87 & 0.249 \\
\hline
\end{tabular}


Table 3. Cont.

\begin{tabular}{cccccccccc}
\hline & $\begin{array}{c}\text { Time } \\
\text { (month) }\end{array}$ & $\boldsymbol{\beta}$ & SE & $\boldsymbol{p}$ & & $\begin{array}{c}\text { Time } \\
\text { (month) }\end{array}$ & $\boldsymbol{\beta}$ & SE & $\boldsymbol{p}$ \\
\hline Methionine & 0 & -2.34 & 3.26 & 0.473 & Citrulline & 0 & -3.28 & 3.06 & 0.284 \\
& 3 & -4.81 & 2.82 & 0.088 & & 3 & -3.58 & 2.18 & 0.101 \\
& 6 & -1.38 & 2.90 & 0.635 & & 6 & -2.28 & 2.35 & 0.332 \\
& 9 & 0.57 & 1.19 & 0.629 & & 9 & -1.91 & 3.02 & 0.528 \\
& 12 & -2.49 & 1.64 & 0.129 & & 12 & 1.17 & 2.26 & 0.603 \\
\hline Glutamic acid & 0 & -3.61 & 12.51 & 0.773 & Homocysteine & 0 & -0.03 & 0.48 & 0.946 \\
& 3 & -20.97 & 14.72 & 0.154 & & 3 & -0.44 & 0.94 & 0.640 \\
& 6 & 5.27 & 10.47 & 0.615 & & 6 & 0.55 & 1.18 & 0.643 \\
& 9 & 6.36 & 11.81 & 0.590 & & 9 & -1.42 & 0.64 & $0.027^{*}$ \\
& 12 & 17.12 & 10.88 & 0.116 & & 12 & -0.69 & 1.09 & 0.524 \\
\hline
\end{tabular}

$\beta$ : regression weight; SE: Standard error; ${ }^{*} p<0.05,{ }^{* *} p<0.01,{ }^{* * *} p<0.05,{ }^{* * * *} p<0.001$.

\subsection{Predictors and Associated Factors of KD Effectiveness}

ROC analysis was applied to identify metabolic parameters that could predict the efficacy of KDT at baseline. The AUCs for C14, C3, and C18 were between 0.79-0.85 when comparing these metabolites at baseline between responders and non-responders (Figure 2A, C14 carnitine, AUC $=0.846, p<0.01 ; \mathrm{C} 3$ carnitine, AUC $=0.835, p<0.005 ; \mathrm{C} 18$ carnitine, AUC $=0.791, p<0.01)$, indicating high predictive ability of KDT effectiveness. This result indicated that a lower baseline $\mathrm{C} 14$ level had the highest predictive ability of KD effectiveness.

(A)

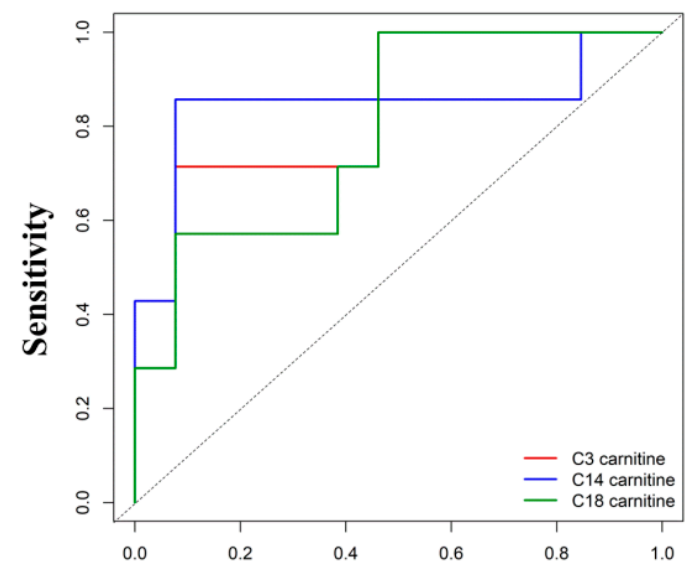

1-Specificity

\begin{tabular}{lccc}
\hline \multicolumn{1}{c}{ Variables } & AUC & Sig. & $\mathbf{9 5 \%}$ CI \\
\hline C14 carnitine & 0.846 & $0.005^{* *}$ & $0.607-1.000$ \\
C3 carnitine & 0.835 & $0.001^{* * *}$ & $0.643-1.000$ \\
C18 carnitine & 0.791 & $0.007^{* *}$ & $0.581-1.000$ \\
C16 carnitine & 0.681 & 0.170 & $0.423-1.000$ \\
C4:OH carnitine & 0.615 & 0.480 & $0.295-0.936$ \\
C14: 1 carnitine & 0.560 & 0.699 & $0.254-0.867$ \\
C16:1 carnitine & 0.550 & 0.783 & $0.197-0.902$ \\
Free carnitine & 0.528 & 0.866 & $0.210-0.846$ \\
C2 carnitine & 0.506 & 0.976 & $0.145-0.866$ \\
\hline
\end{tabular}

(B)

12 months post KD

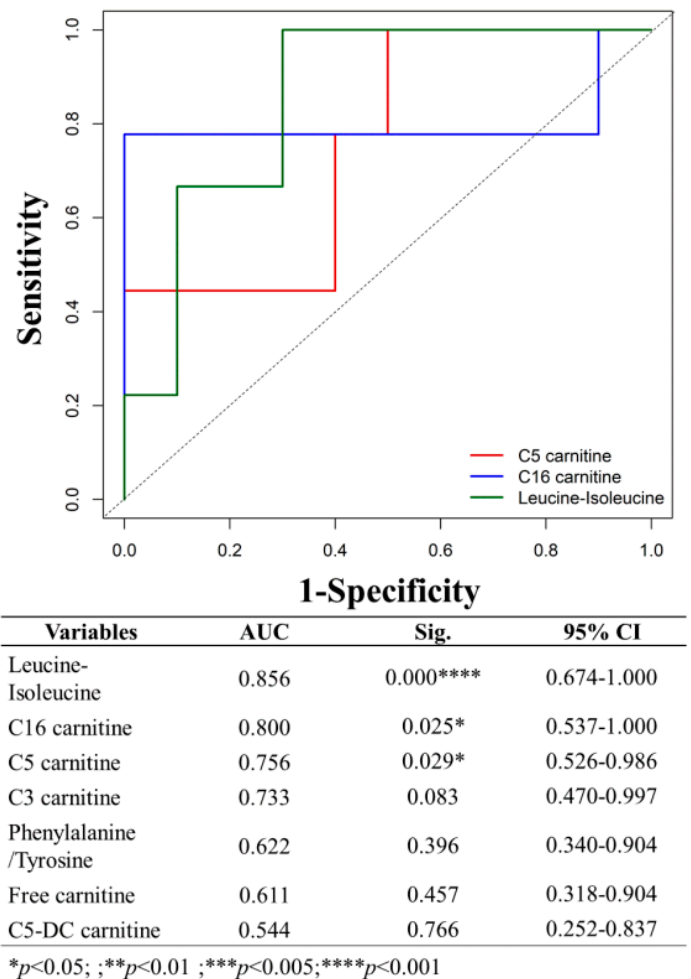

Figure 2. Receiver-operating characteristic (ROC) analysis of the discriminant metabolites between groups. (A) Pre-KD; (B) 12 months post KD. Individual metabolites of significance (solid lines) are shown along with the reference (dotted line). Area under the curve (AUC) values, significance level, and $95 \%$ confidence interval are shown. $\left({ }^{*} p<0.05 ;{ }^{* *} p<0.01\right.$; $\left.{ }^{* * *} p<0.005 ;{ }^{* * * *} p<0.001\right)$. 
After 12 months of KDT, there was a significant decrease in leucine/isoleucine, C16and C 5 carnitines at 12 months as shown in Tables 2 and 3. The AUCs for leucine/isoleucine, C16-and $\mathrm{C} 5$ carnitines were between 0.7 and 0.9 when comparing responders and nonresponders (Figure 2B, leucine/isoleucine, $\mathrm{AUC}=0.856, p<0.001 ; \mathrm{C} 16$ carnitine, $\mathrm{AUC}=0.800$, $p<0.05$; C5 carnitine, AUC $=0.756, p<0.05)$, indicating strong association with KDT effectiveness at 12 months. Leucine-isoleucine had the highest AUC value, followed by C16 carnitine, and then C5 carnitine.

\section{Discussion}

Our results provide evidence that C2 carnitine level significantly increased throughout the diet therapy; otherwise, the long-chain acylcarnitines, C16 and C16:1 levels were significantly decreased after 12 months of KDT. Study subjects had significant lower carnitine levels than baseline values after 12 months of KDT, though carnitine level gradually elevated toward baseline level eventually. It is known that the brain utilizes ketone bodies, especially $\beta \mathrm{HB}$, derived from acetyl-coenzyme A (acetyl-CoA) and acetoacetyl-coenzyme A (acetoacetyl-CoA) produced by $\beta$-oxidation of fatty acids in the mitochondria of the liver [25]. Long-chain fatty acids ( $>12$ carbons) from KDT undergo thio-esterification to CoA and are catalyzed by acyl-CoA synthases (ACS) to form acyl-CoA [26]. Acyl-CoA must be conjugated to carnitine with formation of acyl-carnitines through the action of carnitine palmitoyl transferase I (CPTI), so that it can penetrate mitochondrial membrane. Acyl-carnitines are translocated across the inner mitochondrial membrane by acyl-carnitine translocase (CACT). Carnitine is removed from acyl-carnitine inside the mitochondrion by carnitine palmitoyl transferase II (CPTII), and acyl-CoAs and acetylcarnitine (C2 carnitine) are generated. Acyl-CoAs can enter $\beta$-oxidation in the mitochondrial matrix, finally producing acetyl-CoA, which leads to production of ketone bodies or to entry of citric acid cycle (Figure 3). The higher the efficiency of mitochondrial $\beta$-oxidation or energy metabolism in the liver, the more C2 carnitine is formed from free carnitine, leading to less acumination of long-chain fatty acid and a drop in plasma free carnitine. Thus, the decrement of C16 carnitine and the increment of $\mathrm{C} 2$ carnitine in our result may indicate improvement of mitochondrial functioning and energy metabolism after KD. The clinical practice with regard to carnitine supplement for a drop in free carnitine has huge diversity. We followed the recommendations given by KD expert consensus not routinely supplying carnitine for children with KDT unless they showed biochemical or symptomatic deficiency.

C3-, C5-, C5:OH-, and C4-DC carnitine, derives from ketogenic amino acids, were significantly lower than baseline levels, while $\mathrm{C} 4: \mathrm{OH}$, derived from CoA-ester of the ketone bodies, was significantly higher than baseline values after 12 months of KDT. Since a ketogenic diet provides $80-90 \%$ of calories as lipid, ketogenesis is characterized by a decrease in a protein breakdown and an increase in fat and ketone use [27]. It can provide explanation of opposite alteration of ketogenic amino acids (e.g., C3-C5-C5:OH and C4-DC) and $\mathrm{C} 4: \mathrm{OH}$, the derives of ketone body.

We disclosed C8 and C8/C10 ratio became significantly lower after 12 months of KDT. $\mathrm{C} 8 / \mathrm{C} 10$ ratio is one of the most commonly reported markers in screening for medium-chain acyl-CoA dehydrogenase deficiency (MCADD) [28]. However, none of our study subjects conformed diagnosis of MCADD. Previous report revealed that $\mathrm{C} 8$ has the highest net ketogenic effect compared with other tested oils containing medium-chain triglyceride [23]. We presumed that C8 levels and C8/C10 ratio became lower after KDT may be due to rapidly turnover of $\mathrm{C} 8$ into ketone bodies. Both $\mathrm{C} 14: 1$ and $\mathrm{C} 14: 2$ carnitine levels trended upwards during study period. Elevated C14:1 and C14:2 plasma acylcarnitine after a controlled fast is a diagnostic strategy for very long-chain acyl-coA dehydrogenase (VLCAD) deficiency. However, the elevation of C14:1 and C14:2 plasma acylcarnitines in the setting of prolonged fast and hypoglycemia with induction of lipolysis and generating ketone response is physiologic and not indicative of a diagnosis of VLCAD deficiency [29,30]. Since ketogenic diet is a high fat and low carbohydrate diet which mimics starvation by 
generating ketone bodies, the elevation of $\mathrm{C} 14: 1$ and C14:2 may reflect lipolysis getting progressively increasing as KDT keeping on going.

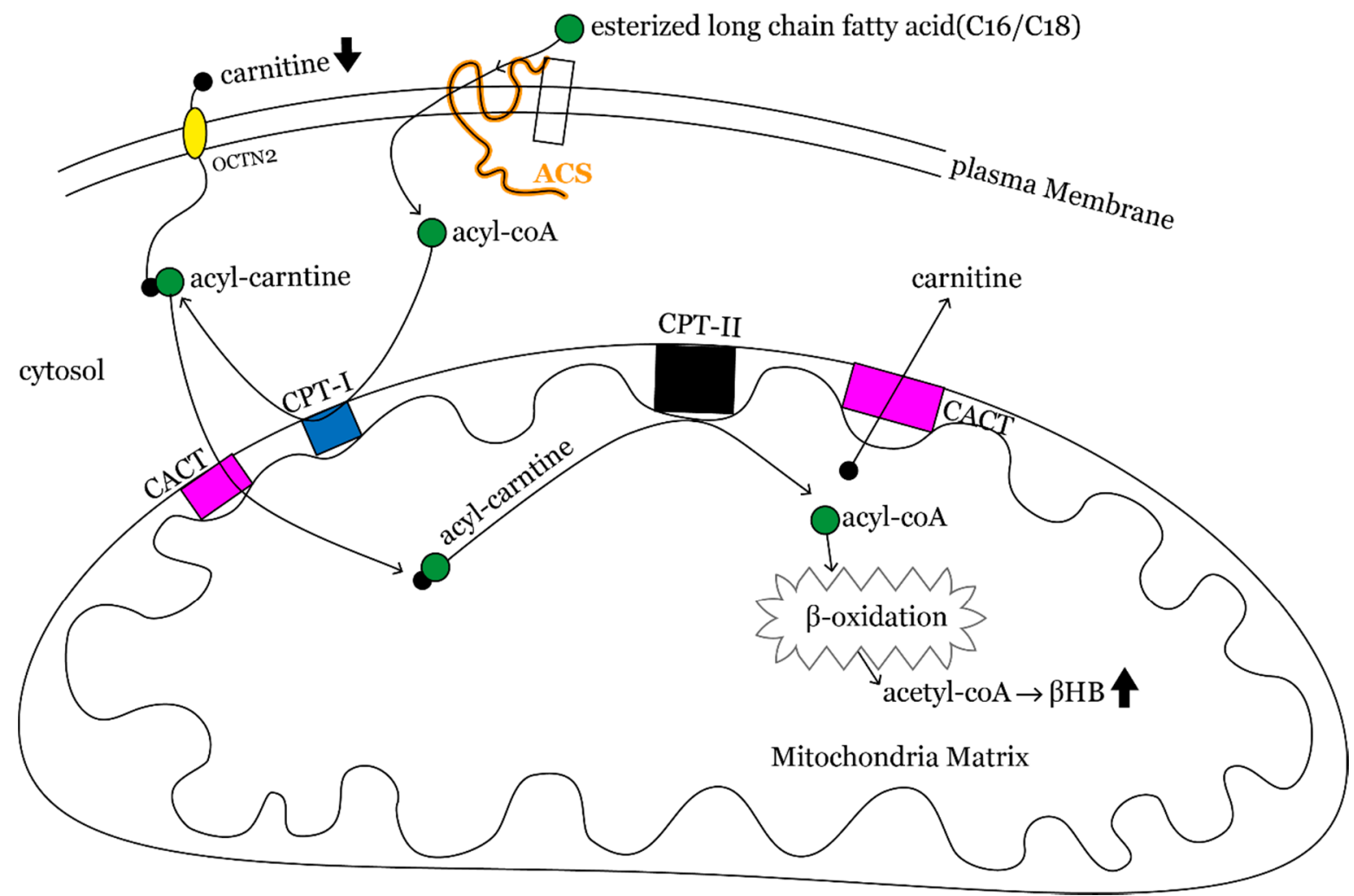

Figure 3. Fatty acid $\beta$-oxidation pathway. Fatty acids (especially long-chain fatty acids) undergo acylation, a process catalyzed by acyl-CoA synthases that traps them in the cytoplasm as acyl-CoA thioesters. Acyl-CoA thioesters then conjugate to carnitine with formation of acylcarnitines through the action of carnitine palmitoyl transferase I. Acylcarnitines are translocated across the inner mitochondrial membrane by acylcarnitine translocase. Carnitine can be removed from acylcarnitine by carnitine palmitoyl transferase II inside the mitochondria, and acyl-CoAs are re-generated. Acyl-CoAs can enter $\beta$-oxidation in the mitochondrial matrix with final products of acetyl-CoA that produce ketone bodies (mainly $\beta \mathrm{HB}$ ) in the liver.

KDT affects not only lipids but also amino acids. There have been several reports on changes in CSF levels of amino acids under KDT [31,32]; however, literature is limited on changes in serum amino acid levels in response to KDT. Millichap found that the only change in serum under KDT was an increased leucine level $[15,33]$. Levels of several amino acids, including phenylalanine, tyrosine, leucine/isoleucine, and methionine, were also significantly lower after 12 months of KDT. Glycine is the only amino acid showing persistently high through study period in our study. Phenylalanine and tyrosine are the substrates of norepinephrine, which is considered one of the mechanisms of the anticonvulsant effect of KDT [34-36]. Consuming phenylalanine and tyrosine to form norepinephrine is presumed to be the possible explanation for their lower levels in our result, and this suggests that norepinephrine is one of the mechanisms of anticonvulsant effect. In addition, methionine is a substrate for biogenesis of carnitine, consumption of carnitine to form acyl-carnitine would lead to consumption of methionine, which explains the decrease in the methionine level $[10,37]$. Although there are reports of KDT being an effective treatment for refractory epilepsy in patients with non-ketotic hyperglycemia [38,39], we suggested that KDT should be avoided in these patients, given the persistently high glycine level based on our results.

We furthermore explored the association between metabolic parameters and the response to KDT. Schoeler et al. [9] reported that $\mathrm{C} 2$ carnitine at baseline was significantly 
higher in responders. However, in our data, responders had significantly lower C2 level at baseline, but its concentration became higher than non-responders after 12 months of KDT. The result was not consistent with the previous report, which demonstrated baseline C2 carnitine is positively associated with greater efficacy of KDT [9]. The most probable explanation for the contradictory results is presumed to be that patients in this study received non-fasting KDT, and the production of acetyl-carnitine to form ketones slowly progressed with time but not in a short time.

Additionally, our data disclosed that C3, C14, C18 at baseline, while C3, C5, C16, and leucine-isoleucine at 12 months are negatively associated with greater efficacy of KDT. Branch-chain amino acids (BACC), such as leucine isoleucine and valine are metabolic fuels that can generate C3- and C5 acylcarnitines. C5 acylcarnitines are comprised of $\alpha$-methylbutyryl and isovalerylcarnitine species, intermediates in mitochondrial isoleucine and leucine catabolism. C3-acylcarnitine reflects the propionyl CoA pool, which is a byproduct of both isoleucine and valine catabolism in the mitochondria. It was reported that accumulation of C3-and C5-acylcarnitines could contribute to incomplete oxidation of fatty acids. Responders and non-responders were different in long-, $\mathrm{C} 3$ and $\mathrm{C} 5$ acylcarnitine indicating they may be distinct in the aspect of mitochondrial functioning. We have more non-responders receiving VPA during study period. The relatively higher long-chain fatty acid, C14, C16, C16:1, C18, C3, and C5 found in non-responders might result from the treatment with VPA. VPA is a fatty acid analog and a substrate for the fatty acid $\beta$ oxidation pathway. VPA combines with CoA or carnitine to form valproyl-CoA and then leads to direct competitive inhibition of carnitine uptake at the transporter site by reducing the availability of free CoA. Mitochondrial depletion of free CoA impairs $\beta$-oxidation of fatty acids and impairs subsequent transport of long-chain fatty acids [40]. Some studies have shown that VPA induces accumulation of long-chain fatty acids (C10-16) in cultured fibroblasts [41] or in humans (C12, C14:1, C16;1, C18:1) [42]. Our results suggested that accumulation of long-chain fatty acids, $\mathrm{C} 3$ and $\mathrm{C} 5$ acylcarnitine is an indicator of impaired fatty acid $\beta$-oxidation. Responders with lower level of long-chain fatty acid and C3 at initiation and long-term management of KDT are assumed to have a better mitochondrial $\beta$-oxidation pathway, which also acted as associated factors for greater effectiveness of KDT in our study.

Responders in this study demonstrated hypocarnitinemia throughout the study. Factors believed to increase the risk of hypocarnitinemia in patients with epilepsy include young age, use of multiple AEDs, and poor nutritional status [43-45]. Although our data indicated a positive correlation between body mass index (BMI) and the free carnitine level (Supplementary Figure S2); however, the tendency of free carnitine and BMI was not similar in this study. Free carnitine level decreased gradually, reaching a nadir at 6 months of therapy, whereas BMI in responders gradually decreased between 9 and 12 months. This finding suggests that hypocarnitinemia in responders cannot be attributed to low BMI. Researchers have found carnitine deficiency in $4-76 \%$ of patients receiving VPA [46-48]. Although more non-responders than responders received VPA as monotherapy or add-on treatment in our study, hypocarnitinemia only occurred in responders. Therefore, treatment with VPA could not be a reason for hypocarnitinemia in patients on KDT in this study. There is another biochemical reason that can explain KDT-related hypocarnitinemia. We presumed that responders possessed a better functioning mitochondrial $\beta$-oxidation pathway, resulting in consuming plasma free carnitine, which provided the best explanation of hypocarnitinemia in responders.

Like previous researches on KDT, this study has some limitations, including a small sample size, lack of blinding or randomization, lack of a control group, and lack of age stratification. The human metabolome is influenced by a number of endogenous factors, such as age, sex, and BMI [49-52], so that age stratification is mandatory if we can extend the study numbers. Blinding is difficult in KDT research for obvious reasons. The effectiveness of KDT in patients with refractory epilepsy is well documented, so it would be ethically to randomize patients to study groups. Patients with refractory epilepsy account for only 
$30 \%$ of all cases of pediatric epilepsy, so it is inevitable that those receiving KDT are a small population. Finally, given the small sample size, we could not stratify our patients by age.

\section{Conclusions}

The novel finding of this study is that C3-, C5-, C5:OH-, and C4-DC decreased, and $\mathrm{C} 4 \mathrm{OH}$ increased significantly at 12 months of KDT indicated decreasing glucose breakdown with increasing ketosis on long-term KDT. We also suggested that KDT should be avoided in patients with non-ketotic hyperglycemia, given the persistently high glycine level based on our results. Transient hypocarnitinemia was found during 9 months of KDT but was asymptomatic and resolved eventually, so that there is no need for carnitine supplementation. Our results also afford a new insight into the metabolic parameters that predict the response to KDT. The lower C3, C14, C18 at baseline could predict the efficacy of KDT, whereas lower C5, C16, and Ile/Leu level were factors associated with the effectiveness of long-term KDT. The results indicated the better mitochondrial ßoxidation function is associated with greater KDT response.

Supplementary Materials: The following are available online at https:/ / www.mdpi.com/2072-6 $643 / 13 / 1 / 21 / s 1$. Figure S1: The correlation of free carnitine between $\beta H B$, and seizure reduction rate. (A) The plasma free carnitine was negatively correlated with $\beta$ HB level $(\rho=0.478, p=0.028)$. (B) Neither $\beta \mathrm{HB}$ nor free carnitine level was correlated with seizure reduction rate $(\beta \mathrm{HB}$ vs. seizure reduction rate, $\rho=-0.427, p=0.252$; free carnitine vs. seizure reduction rate, $\rho=-0.109, p=0.781$ ), Figure S2: The plasma free carnitine was positively correlated with body mass index by Spearman zero order correlation $(\rho=0.478, p<0.05)$. Table S1: Demographic data of 22 study subjects. Table S2: The clinical characteristics of responders and non-responders. Table S3: The raw data of metabolic parameters in responders and non-responders for serial follow-up.

Author Contributions: Conceptualization, P.-L.H., C.C. and Y.-J.L.; methodology, P.-L.H., J.-L.L. and Y.-J.L.; validation, C.C., J.-L.L., P.-L.H. and Y.-J.L.; formal analysis, J.-L.L., K.-Y.H. and T.-Y.H.; investigation, P.-L.H. and Y.-J.L.; resources, P.-L.H.; data curation, K.-Y.H., T.-Y.H., M.-H.H. and H.-C.K.; writing—original draft preparation, P.-L.H., Y.-J.L., K.-Y.H. and T.-Y.H.; writing—review and editing, M.-H.H., H.-C.K., P.-L.H. and Y.-J.L.; supervision, P.-L.H., C.C. and Y.-J.L.; project administration, P.-L.H. and Y.-J.L.; funding acquisition, P.-L.H. All authors have read and agreed to the published version of the manuscript.

Funding: This research was funded by Research Support Scheme of Chang Gung Memorial Hospital (CMRPG8G0951).

Acknowledgments: This study was supported by grants from the Research Support Scheme of Chang Gung Memorial Hospital (CMRPG8G0951). We thank the Biostatistics Center of Kaohsiung Chang Gung Memorial Hospital for its assistance with the statistical analysis. The funding source played no role in the study design, collection, analysis, interpretation of the data, writing of the manuscript, or the decision to submit the manuscript for publication. We also thank Ting-Fang Cheng for her skillful assistance with data preparation.

Conflicts of Interest: None of the authors has any conflicts of interest to disclose. We confirm that we have read the Journal's position on issues involved in ethical publication and affirm that this report is consistent with those guidelines.

\section{References}

1. Vining, E.P.; Freeman, J.M.; Ballaban-Gil, K.; Camfield, C.S.; Camfield, P.R.; Holmes, G.L.; Shinnar, S.; Shuman, R.; Trevathan, E.; Wheless, J.W. A multicenter study of the efficacy of the ketogenic diet. Arch. Neurol. 1998, 55, 1433-1437. [CrossRef] [PubMed]

2. Freeman, J.M.; Vining, E.P.; Pillas, D.J.; Pyzik, P.L.; Casey, J.C.; Kelly, L.M. The efficacy of the ketogenic diet-1998: A prospective evaluation of intervention in 150 children. Pediatrics 1998, 102, 1358-1363. [CrossRef] [PubMed]

3. Hassan, A.M.; Keene, D.L.; Whiting, S.E.; Jacob, P.J.; Champagne, J.R.; Humphreys, P. Ketogenic diet in the treatment of refractory epilepsy in childhood. Pediatr. Neurol. 1999, 21, 548-552. [CrossRef]

4. Katyal, N.G.; Koehler, A.N.; McGhee, B.; Foley, C.M.; Crumrine, P.K. The ketogenic diet in refractory epilepsy: The experience of Children's Hospital of Pittsburgh. Clin. Pediatr. 2000, 39, 153-159. [CrossRef] [PubMed]

5. Wlodarek, D. Role of Ketogenic Diets in Neurodegenerative Diseases (Alzheimer's Disease and Parkinson's Disease). Nutrients 2019, 11, 169. [CrossRef] 
6. Kossoff, E.H.; Zupec-Kania, B.A.; Amark, P.E.; Ballaban-Gil, K.R.; Bergqvist, A.G.C.; Blackford, R.; Buchhalter, J.R.; Caraballo, R.H.; Cross, J.H.; Dahlin, M.G.; et al. Optimal clinical management of children receiving the ketogenic diet: Recommendations of the International Ketogenic Diet Study Group. Epilepsia 2009, 50, 304-317. [CrossRef]

7. Schoeler, N.E.; Cross, J.H.; Sander, J.W.; Sisodiya, S.M. Can we predict a favourable response to Ketogenic Diet Therapies for drug-resistant epilepsy? Epilepsy Res. 2013, 106, 1-16. [CrossRef]

8. Meidenbauer, J.J.; Mukherjee, P.; Seyfried, T.N. The glucose ketone index calculator: A simple tool to monitor therapeutic efficacy for metabolic management of brain cancer. Nutr. Metab. 2015, 12, 12. [CrossRef]

9. Schoeler, N.E.; Bell, G.; Yuen, A.; Kapelner, A.D.; Heales, S.J.R.; Cross, J.H.; Sisodiya, S. An examination of biochemical parameters and their association with response to ketogenic dietary therapies. Epilepsia 2017, 58, 893-900. [CrossRef]

10. Berry-Kravis, E.; Booth, G.; Sanchez, A.C.; Woodbury-Kolb, J. Carnitine levels and the ketogenic diet. Epilepsia 2001, 42, $1445-1451$. [CrossRef]

11. Boison, D. New insights into the mechanisms of the ketogenic diet. Curr. Opin. Neurol. 2017, 30, 187-192. [CrossRef] [PubMed]

12. Freeman, J.M.; Kossoff, E.H.; Hartman, A.L. The ketogenic diet: One decade later. Pediatrics 2007, 119, 535-543. [CrossRef] [PubMed]

13. D'Andrea Meira, I.; Romao, T.T.; Pires do Prado, H.J.; Kruger, L.T.; Pires, M.E.P.; da Conceicao, P.O. Ketogenic Diet and Epilepsy: What We Know So Far. Front. Neurosci. 2019, 13, 5. [CrossRef] [PubMed]

14. Sariego-Jamardo, A.; Garcia-Cazorla, A.; Artuch, R.; Castejón, E.; García-Arenas, D.; Molero-Luis, M.; Ormazábal, A.; Sanmartí, F.X.; Molero, M. Efficacy of the Ketogenic Diet for the Treatment of Refractory Childhood Epilepsy: Cerebrospinal Fluid Neurotransmitters and Amino Acid Levels. Pediatr. Neurol. 2015, 53, 422-426. [CrossRef]

15. Yudkoff, M.; Daikhin, Y.; Melo, T.M.; Nissim, I.; Sonnewald, U.; Nissim, I. The ketogenic diet and brain metabolism of amino acids: Relationship to the anticonvulsant effect. Annu. Rev. Nutr. 2007, 27, 415-430. [CrossRef]

16. Yudkoff, M.; Daikhin, Y.; Nissim, I.; Lazarow, A.; Nissim, I. Ketogenic diet, brain glutamate metabolism and seizure control. Prostaglandins Leukot Essent. Fat. Acids 2004, 70, 277-285. [CrossRef]

17. Jirapinyo, P.; Kankirawatana, P.; Densupsoontorn, N.; Thamonsiri, N.; Wongarn, R. High plasma branched-chain amino acids:aromatic amino acids ratio in children on the ketogenic diet: A mechanism in controlling epilepsy. J. Med. Assoc. Thai. 2004, 87, 432-437.

18. Kwan, P.; Arzimanoglou, A.; Berg, A.T.; Brodie, M.J.; Hauser, W.A.; Mathern, G.; Moshé, S.L.; Perucca, E.; Wiebe, S.; French, J. Definition of drug resistant epilepsy: Consensus proposal by the ad hoc Task Force of the ILAE Commission on Therapeutic Strategies. Epilepsia 2010, 51, 1069-1077. [CrossRef]

19. Yala, A.; Lehman, C.; Schuster, T.; Portnoi, T.; Barzilay, R. A Deep Learning Mammography-based Model for Improved Breast Cancer Risk Prediction. Radiology 2019, 292, 60-66. [CrossRef]

20. Soeters, M.R.; Serlie, M.J.; Sauerwein, H.P.; Duran, M.; Ruiter, J.P.; Kulik, W.; Ackermans, M.T.; Minkler, P.E.; Hoppel, C.L.; Wanders, R.J.A.; et al. Characterization of D-3-hydroxybutyrylcarnitine (ketocarnitine): An identified ketosis-induced metabolite. Metabolism 2012, 61, 966-973. [CrossRef]

21. Rinaldo, P.; Cowan, T.M.; Matern, D. Acylcarnitine profile analysis. Genet. Med. 2008, 10, 151-156. [CrossRef] [PubMed]

22. Mihalik, S.J.; Goodpaster, B.H.; Kelley, D.E.; Chace, D.H.; Vockley, J.; Toledo, F.G.; Delany, J.P. Increased levels of plasma acylcarnitines in obesity and type 2 diabetes and identification of a marker of glucolipotoxicity. Obesity 2010, 18, 1695-1700. [CrossRef] [PubMed]

23. Vandenberghe, C.; St-Pierre, V.; Pierotti, T.; Fortier, M.; Castellano, C.A.; Cunnane, S.C. Tricaprylin Alone Increases Plasma Ketone Response More Than Coconut Oil or Other Medium-Chain Triglycerides: An Acute Crossover Study in Healthy Adults. Curr. Dev. Nutr. 2017, 1, e000257. [CrossRef] [PubMed]

24. De Vivo, D.C.; Bohan, T.P.; Coulter, D.L.; Dreifuss, F.E.; Greenwood, R.S.; Nordli, D.R.; Shields, W.D.; Stafstrom, C.E.; Tein, I. L-carnitine supplementation in childhood epilepsy: Current perspectives. Epilepsia 1998, 39, 1216-1225. [CrossRef]

25. Longo, N.; Amat di San Filippo, C.; Pasquali, M. Disorders of carnitine transport and the carnitine cycle. Am. J. Med. Genet. C Semin. Med. Genet. 2006, 142C, 77-85. [CrossRef]

26. Watkins, P.A.; Maiguel, D.; Jia, Z.; Pevsner, J. Evidence for 26 distinct acyl-coenzyme A synthetase genes in the human genome. J. Lipid Res. 2007, 48, 2736-2750. [CrossRef]

27. Swink, T.D.; Vining, E.P.; Freeman, J.M. The ketogenic diet: 1997. Adv. Pediatr. 1997, 44, 297-329.

28. Couce, M.L.; Sánchez-Pintos, P.; Diogo, L.; Leão-Teles, E.; Martins, E.; Santos, H.; Couce, M.L.; Delgado-Pecellín, C.; Castiñeiras, D.; Cocho, J.A.; et al. Newborn screening for medium-chain acyl-CoA dehydrogenase deficiency: Regional experience and high incidence of carnitine deficiency. Orphanet J. Rare Dis. 2013, 8, 102. [CrossRef]

29. Costa, C.C.; de Almeida, I.T.; Jakobs, C.; Poll-The, B.T.; Duran, M. Dynamic changes of plasma acylcarnitine levels induced by fasting and sunflower oil challenge test in children. Pediatr. Res. 1999, 46, 440-444. [CrossRef]

30. Hoppel, C.L.; Genuth, S.M. Carnitine metabolism in normal-weight and obese human subjects during fasting. Am. J. Physiol. 1980, 238, E409-E415. [CrossRef]

31. Yudkoff, M.; Daikhin, Y.; Nissim, I.; Lazarow, A.; Nissim, I. Ketogenic diet, amino acid metabolism, and seizure control. J. Neurosci. Res. 2001, 66, 931-940. [CrossRef] [PubMed]

32. Yudkoff, M.; Daikhin, Y.; Nissim, I.; Horyn, O.; Lazarow, A.; Luhovyy, B.; Wehrli, S. Response of brain amino acid metabolism to ketosis. Neurochem. Int. 2005, 47, 119-128. [CrossRef] [PubMed] 
33. Millichap, J.G. CSF Amino Acids, Pterins and Mechanism of the Ketogenic Diet. Pediatr. Neurol. Briefs 2015, 29, 79. [CrossRef]

34. Lima, P.A.; Sampaio, L.P.; Damasceno, N.R. Neurobiochemical mechanisms of a ketogenic diet in refractory epilepsy. Clinics 2014, 69, 699-705. [CrossRef]

35. Szot, P.; Weinshenker, D.; Rho, J.M.; Storey, T.W.; Schwartzkroin, P.A. Norepinephrine is required for the anticonvulsant effect of the ketogenic diet. Brain Res. Dev. Brain Res. 2001, 129, 211-214. [CrossRef]

36. Weinshenker, D. The contribution of norepinephrine and orexigenic neuropeptides to the anticonvulsant effect of the ketogenic diet. Epilepsia 2008, 49 (Suppl. 8), 104-107. [CrossRef]

37. Liebhaber, G.; Pascher, B.; Gempel, K.; Baumeister, F.A. Asymptomatic carnitine depletion on ketogenic diet in patients with pharmacoresistant epilepsies. Klin. Padiatr. 2006, 218, 260-263. [CrossRef]

38. Kava, M.P.; Robertson, A.; Greed, L.; Balasubramaniam, S. Ketogenic diet, a potentially valuable therapeutic option for the management of refractory epilepsy in classical neonatal nonketotic hyperglycinemia: A case report. Eur. J. Clin. Nutr. 2019, 73, 961-965. [CrossRef]

39. Cusmai, R.; Martinelli, D.; Moavero, R.; Vici, C.D.; Vigevano, F.; Castana, C.; Elia, M.; Bernabei, S.; Bevivino, E. Ketogenic diet in early myoclonic encephalopathy due to non ketotic hyperglycinemia. Eur. J. Paediatr. Neurol. 2012, 16, 509-513. [CrossRef]

40. Lheureux, P.E.; Penaloza, A.; Zahir, S.; Gris, M. Science review: Carnitine in the treatment of valproic acid-induced toxicity-What is the evidence? Crit. Care 2005, 9, 431-440. [CrossRef]

41. Silva, M.F.; Jakobs, C.; Duran, M.; de Almeida, I.T.; Wanders, R.J. Valproate induces in vitro accumulation of long-chain fatty acylcarnitines. Mol. Genet. Metab. 2001, 73, 358-361. [CrossRef]

42. Nakajima, Y.; Ito, T.; Maeda, Y.; Ichiki, S.; Kobayashi, S.; Ando, N.; Hussein, M.H.; Kurono, Y.; Sugiyama, N.; Togari, H. Evaluation of valproate effects on acylcarnitine in epileptic children by LC-MS/MS. Brain Dev. 2011, 33, 816-823. [CrossRef]

43. Riva, R.; Albani, F.; Gobbi, G.; Santucci, M.; Baruzzi, A. Carnitine disposition before and during valproate therapy in patients with epilepsy. Epilepsia 1993, 34, 184-187. [CrossRef]

44. Coulter, D.L. Carnitine deficiency in epilepsy: Risk factors and treatment. J. Child Neurol. 1995, 10, S32-S39. [CrossRef]

45. Hug, G.; McGraw, C.A.; Bates, S.R.; Landrigan, E.A. Reduction of serum carnitine concentrations during anticonvulsant therapy with phenobarbital, valproic acid, phenytoin, and carbamazepine in children. J. Pediatr. 1991, 119, 799-802. [CrossRef]

46. Opala, G.; Winter, S.; Vance, C.; Vance, H.; Hutchison, H.T.; Linn, L.S. The effect of valproic acid on plasma carnitine levels. Am. J. Dis. Child 1991, 145, 999-1001. [CrossRef]

47. Hiraoka, A.; Arato, T.; Tominaga, I. Reduction in blood free carnitine levels in association with changes in sodium valproate (VPA) disposition in epileptic patients treated with VPA and other anti-epileptic drugs. Biol. Pharm. Bull. 1997, 20, 91-93. [CrossRef]

48. Beghi, E.; Bizzi, A.; Codegoni, A.M.; Trevisan, D.; Torri, W. Valproate, carnitine metabolism, and biochemical indicators of liver function. Collaborative Group for the Study of Epilepsy. Epilepsia 1990, 31, 346-352. [CrossRef]

49. Auro, K.; Joensuu, A.; Fischer, K.; Kettunen, J.; Salo, P.; Mattsson, H.; Niironen, M.; Kaprio, J.; Eriksson, J.G.; Lehtimäki, T.; et al. A metabolic view on menopause and ageing. Nat. Commun. 2014, 5, 4708. [CrossRef] [PubMed]

50. Kochhar, S.; Jacobs, D.M.; Ramadan, Z.; Berruex, F.; Fuerholz, A.; Fay, L.B. Probing gender-specific metabolism differences in humans by nuclear magnetic resonance-based metabonomics. Anal. Biochem. 2006, 352, 274-281. [CrossRef]

51. Mittelstrass, K.; Ried, J.S.; Yu, Z.; Krumsiek, J.; Gieger, C.; Prehn, C.; Roemisch-Margl, W.; Polonikov, A.; Peters, A.; Theis, F.J.; et al. Discovery of sexual dimorphisms in metabolic and genetic biomarkers. PLoS Genet. 2011, 7, e1002215. [CrossRef]

52. Slupsky, C.M.; Rankin, K.N.; Wagner, J.; Fu, H.; Chang, D.; Weljie, A.M.; Saude, E.J.; Lix, B.; Adamko, D.J.; Shah, S.; et al. Investigations of the effects of gender, diurnal variation, and age in human urinary metabolomic profiles. Anal. Chem. 2007, 79, 6995-7004. [CrossRef] 